\title{
Neonatal Anesthesia
}

\section{Jerrold Lerman (Editor). Springer Science+Business Media 2015. ISBN 978-1-4419-6040-5}

\author{
Carolyne Montgomery, MD $\cdot$ J. Mark Ansermino, MBBCh
}

Received: 9 July 2015/Accepted: 21 July 2015/Published online: 14 August 2015

(C) Canadian Anesthesiologists' Society 2015

A textbook dedicated to neonatal anesthesia is a welcome addition to an academic pediatric anesthesiology library. Prior to this 2015 publication by Dr. Leman and his team, readers relied on work published in the late 1980s and specialty chapters in the standard pediatric anesthesiology textbooks. The unique anatomical, physiological, and pharmacological differences encountered in the neonatal period should rightly warrant a text dedicated to this subset of patients.

The challenge in devoting an entire 458-page textbook to neonatal anesthesia is finding and synthesizing specific scientific and clinical material relating to this challenging specialty rather than to the realm of infants and children. The highly published and renowned international team of authors includes thought leaders in many specific areas of pediatric anesthesia, including a neonatal surgeon, a neonatologist, and several pediatric intensivists.

Overall, the book has many outstanding chapters that have been thoughtfully crafted by leading researchers in specific fields. The illustrations are colourful and numerous, and the reference lists are typically very extensive. There are certain essential chapters in this 17-chapter text, specifically, chapter 2 (physiology and development), 3 (pharmacology), 5 (airway management), 10 (thoracoabdominal and general surgery), 12 (neonatal cardiac surgery), and 13 (anesthesia outside the operating room). While there is some content overlap in some of these chapters, the material is primarily specific and up to date. The chapters Regional Anesthesia and Pain

C. Montgomery, MD · J. M. Ansermino, MBBCh $(\bowtie)$

Pediatric Anesthesia, University of British Columbia,

Vancouver, BC, Canada

e-mail: mansermino@cw.bc.ca
Assessment and Management provide focused reviews of the infant and neonatal literature.

In many of the remaining chapters, the authors are challenged by the limited specific neonatal data readily available in the global academic literature, and thus, there is a lot of reiteration of content that would be found in a major pediatric anesthesia text. As with any textbook, many of the chapters are based on current clinical practice and expert opinion rather than on scientific evidence. This should not be considered a limitation but rather a challenge to researchers to provide the evidence that supports or changes clinical practice.

The chapter Anesthesia Outside the Operating Room addresses surgery in the neonatal intensive care unit, including radiology. This area of neonatal anesthetic practice has increased significantly over the last few years; however, as many neonatal cardiac procedures are performed in the cardiac catheterization lab, a separate chapter on this area would have been helpful.

One of the most relevant topics in neonatal anesthesia that has raised significant public concern is the issue of anesthetic neurotoxicity. This subject, undoubtedly one of the most concerning issues for anesthesiologists and parents, is addressed in at least two chapters, but unfortunately, the content is rather poorly structured with a long list of references and no clear recommendations. Other areas that would have been of interest to explore in greater detail include antenatal fetal surgery, perinatology, neonatal resuscitation, acute and longer term palliative care of the neonate, thermal homeostasis, and gestational age outcomes. In particular, the readability of the chapter on neonatal ventilation would have benefitted from more graphic support and an expansion on the details regarding high frequency techniques such jet and oscillatory ventilation. 
These reviewers were given a hardcover textbook and were unable to locate or review an electronic version. A searchable electronic format with technical videos would be invaluable, particularly for any neonatal procedures such as vascular access and regional anesthesia where video teaching has become the standard. Regardless, this book is an essential addition to any pediatric anesthesiology library in any organization that provides care to neonates. This book is an excellent resource, not only for anesthesiologists at every level of training and expertise but also for other professionals who provide postoperative care, administer sedative drugs, control the airway, and undertake procedures in newborn infants. We encourage the publishers to support the authors in creating a searchable and accessible point-ofcare electronic format for the next edition.

Conflicts of interest None declared. 